OHSTPY-HEP-T-04-005

hep-th/0405017

\title{
Dual geometries for a set of 3-charge microstates
}

\author{
Stefano Giusto, Samir D. Mathur and Ashish Saxena \\ Department of Physics, \\ The Ohio State University, \\ Columbus, OH 43210, USA
}

\begin{abstract}
We construct a set of extremal D1-D5-P solutions, by taking appropriate limits in a known family of nonextremal 3-charge solutions. The extremal geometries turn out to be completely smooth, with no horizon and no singularity. The solutions have the right charges to be the duals of a family of CFT microstates which are obtained by spectral flow from the NS vacuum.
\end{abstract}

giusto@mps.ohio-state.edu,mathur@mps.ohio-state.edu, ashish@pacific.mps.ohio-state.edu 


\section{Introduction}

In the traditional picture of a black hole, infalling matter settles into a central singularity while Hawking radiation emerges at the horizon. Due to the large separation between the horizon and the singularity the radiation is insensitive to the detailed state of the matter that made the hole, and we get information loss [1].

Some computations in string theory suggest that the black hole interior is quite different; instead of 'empty space with a central singularity' we have a 'fuzzball' with state information distributed throughout the interior of the horizon. It was shown in [2] that due to the phenomenon of 'fractionation' the effective excitations of a D1-D5-P bound state are very light, and in fact extend to a distance of order the horizon radius.

In [3, 4 the 2-charge extremal D1-D5 system was studied. The 'naive' geometry of D1 and D5 branes is pictured in Fig.1(a); we have flat space at $r \rightarrow \infty$ and a singularity at $r=0$. But the CFT analysis implies that the Ramond (R) ground state of the D1-D5 system is highly degenerate, with entropy $S=2 \sqrt{2} \sqrt{n_{1} n_{5}}$. In [3] the geometries dual to these states were constructed. It was found that the naive metric did not arise from any of the microstates; instead all states yielded geometries that were 'capped' smoothly before reaching $r=0 .{ }^{1}$ No individual geometry has a horizon or singularity but if we draw a surface to bound the area where these geometries differ significantly from the naive geometry then from the area $A$ of this surface we find

$$
\frac{A}{4 G} \sim 2 \sqrt{2} \sqrt{n_{1} n_{5}}
$$

The radius of this surface is $\sim\left(n_{1} n_{5}\right)^{\frac{1}{6}}$ times the Planck length or the string length (the dilaton is bounded, so $l_{p} \sim l_{s}$ ). Thus we see that the D1-D5 bound state 'swells' up to a radius that increases with the charges, and which is such that the bounding surface constructed above bears a Bekenstein type relation to the count of states.

If we have three charges - D1, D5 and momentum $\mathrm{P}$ - then the 'naive' geometry is an extreme Reissner-Nordstrom type black hole. This geometry has a horizon at $r=0$, and continues to a region $r<0$ which contains a singularity (Fig.1(c)). The area of the horizon gives

$$
\frac{A}{4 G}=2 \pi \sqrt{n_{1} n_{5} n_{p}}
$$

and this exactly equals the microscopic entropy obtained from a count of D1-D5-P ground states [6]. But based on the results above we are led to ask if the individual states are described by geometries that 'cap off' before reaching $r=0$ as in Fig.1(d). For three charges the radius of the 'throat' asymptotes to a constant as we go down the throat, so the area $A$ obtained at the dashed line in Fig.1(d) will give (1.2). Thus the nontrivial question in this case is whether the geometries dual to 3-charge microstates are like Fig.1(c) (with a horizon and singularity inside the horizon) or whether some effects

\footnotetext{
${ }^{1}$ The construction in [3] had an apparent singularity along a closed curve in the 'cap', but it was shown in [5] that this was just a coordinate singularity.
} 
destroy this naive expectation before we reach $r=0$. Note that in the 3-charge case (unlike the 2-charge case) we do not expect the generic state to be well-described by a classical geometry; quantum fluctuations can be large. But there would still be special cases that are in fact well described by a classical metric, and we can gain insight by constructing these explicitly.

In [7] a perturbation was constructed on an extremal 2-charge D1-D5 state that added one unit of $\mathrm{P}$ charge. The equation for linear perturbations was solved to give a regular, normalizable excitation in the limits of small $r$ and large $r$, and the solutions were shown to agree to several orders in the region of overlap. This indicated that at least this particular 3-charge state was smoothly 'capped' as in Fig.1(d), and did not have a horizon or singularity like Fig.1(c).

In the present paper we obtain exact geometries dual to a set of D1-D5-P microstates. These geometries will again turn out to be capped as in Fig.1(d). The microstates are not generic 3-charge states; in particular they have a significant amount of rotation. But the construction does support the general conjecture that all configurations must suffer modifications before reaching $r=0$ and forming a horizon.

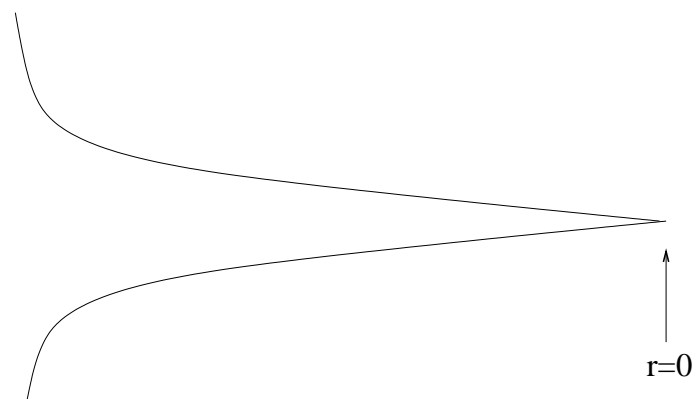

(a)

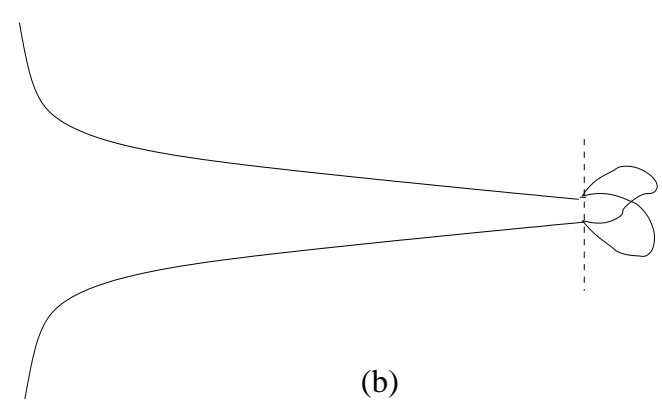

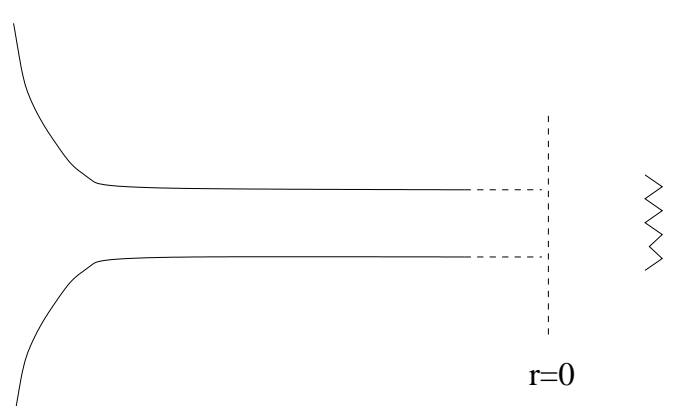

(c)

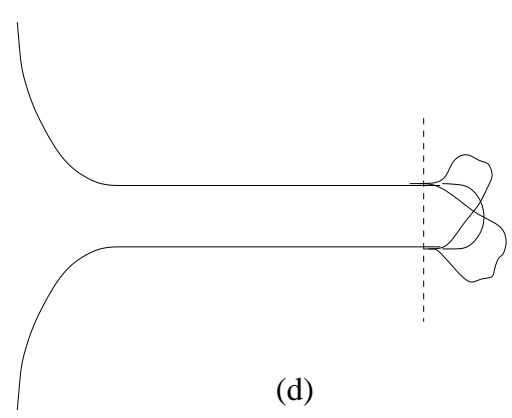

Figure 1: (a) Naive geometry of 2-charge D1-D5. (b) Actual geometries of 2-charge D1-D5; the area of the surface shown by the dashed line gives $\frac{A}{4 G} \sim \sqrt{n_{1} n_{5}}$. (c) Naive geometry of 3 -charge D1-D5-P; there is a horizon at $r=0$ and a singularity past the horizon. (d) Expected geometries for D1-D5-P; the area at the dashed line will give $\frac{A}{4 G}=2 \pi \sqrt{n_{1} n_{5} n_{p}}$.

While we were finishing this work the paper [8] appeared, which also constructed 
similar metrics by an interesting though different method based on 9. If we set the D1 and D5 charges equal in our solution $\left(Q_{1}=Q_{5}\right)$ then the dilaton vanishes, and we obtain solutions that look (locally) like the solutions in 8 . There does appear to be a difference however in the way the final parameters are set in the solution, so that the values of the conserved quantities like angular momenta in [8] appear to be different from the ones that we have. We comment briefly on these issues near the end of our paper.

\section{The CFT states}

\subsection{The D1-D5 CFT}

We take IIB string theory compactified to $M_{4,1} \times S^{1} \times T^{4}$. Let $y$ be the coordinate along $S^{1}$ with

$$
0 \leq y<2 \pi R
$$

The $T^{4}$ is described by 4 coordinates $z_{1}, z_{2}, z_{3}, z_{4}$, and the noncompact space is spanned by $t, x_{1}, x_{2}, x_{3}, x_{4}$. We wrap $n_{1}$ D1 branes on $S^{1}$, and $n_{5}$ D5 branes on $S^{1} \times T^{4}$. Let $N=n_{1} n_{5}$. The bound state of these branes is described by a $1+1$ dimensional sigma model, with base space $(y, t)$ and target space a deformation of the orbifold $\left(T^{4}\right)^{N} / S_{N}$ (the symmetric product of $N$ copies of $T^{4}$ ). The CFT has $\mathcal{N}=4$ supersymmetry, and a moduli space which preserves this supersymmetry. It is conjectured that in this moduli space we have an 'orbifold point' where the target space is just the orbifold $\left(T^{4}\right)^{N} / S_{N}$ [10].

The CFT with target space just one copy of $T^{4}$ is described, at the orbifold point, by 4 real bosons $X^{1}, X^{2}, X^{3}, X^{4}$ (which arise from the 4 directions $z_{1}, z_{2}, z_{3}, z_{4}$ ), 4 real left moving fermions $\psi^{1}, \psi^{2}, \psi^{3}, \psi^{4}$ and 4 real right moving fermions $\bar{\psi}^{1}, \bar{\psi}^{2}, \bar{\psi}^{3}, \bar{\psi}^{4}$. The central charge is $c=6$. The complete theory with target space $\left(T^{4}\right)^{N} / S_{N}$ has $N$ copies of this $c=6 \mathrm{CFT}$, with states that are symmetrized between the $N$ copies. The orbifolding also generates 'twist' sectors, which are created by twist operators $\sigma_{n}$; for a detailed construction of the $\sigma_{n}$ in this theory see [11, 12. We will not be working with the twist sectors in this paper - all the states we construct are in the 'untwisted sector'.

The rotational symmetry of the noncompact directions $x_{1} \ldots x_{4}$ gives a symmetry $s o(4) \approx s u(2)_{L} \times s u(2)_{R}$. The left fermions $\psi^{i}$ carry spin $\frac{1}{2}$ under $s u(2)_{L}$ and the right fermions $\bar{\psi}^{i}$ carry spin $\frac{1}{2}$ under $s u(2)_{R}$. The 'charge' of a state is given by the quantum numbers $(j, \bar{j})=\left(j_{L}^{3}, j_{R}^{3}\right)$.

Consider one copy of the $c=6 \mathrm{CFT}$, and look at the left sector. The fermions can be antiperiodic around $y$ (NS sector) or periodic (R sector). The NS vacuum has $h=j=0$. The NS sector states can be mapped to $\mathrm{R}$ sector states by 'spectral flow' 13], under which the conformal dimensions and charges change as

$$
\begin{aligned}
h^{\prime} & =h-\alpha q+\alpha^{2} \frac{c}{24} \\
q^{\prime} & =q-\alpha \frac{c}{12}
\end{aligned}
$$


Setting $\alpha=1$ gives the flow from the NS sector to the $\mathrm{R}$ sector, and we can see that under this flow chiral primaries of the NS sector (which have $h=q$ ) map to Ramond ground states with $h=\frac{c}{24}$.

The field theory on the D1-D5 branes system is in the R sector. This follows from the fact that the branes are solitons of the gravity theory, and the fermions on the branes are induced from fermions on the bulk. The latter are periodic around the $S^{1}$; choosing antiperiodic boundary conditions would give a nonvanishing vacuum energy and disallow the flat space solution that we have assumed at infinity. The geometries constructed in [3] described gravity duals of the $\mathrm{R}$ ground states of the CFT.

If we set $\alpha=2$ in (2.3) then we return to the NS sector, and setting $\alpha=3$ brings us again to the $\mathrm{R}$ sector. More generally, the choice

$$
\alpha=2 n+1, \quad n \text { integer }
$$

brings us to the $\mathrm{R}$ sector. From (2.3) we see that if we start with a $\mathrm{R}$ ground state obtained at $\alpha=1$, then we get another $\mathrm{R}$ ground state $\left(h=\frac{c}{24}\right)$ at $\alpha=-1$, but for $\alpha=3,5,7 \ldots$ and $\alpha=-3,-5,-7 \ldots$ we get excited states of the $\mathrm{R}$ sector.

\subsection{The states we consider}

We will look at states where we do a spectral flow (2.4) on the left sector, and a spectral flow with $\alpha=1$ on the right sector. Thus the right movers will be in an $\mathrm{R}$ ground state, and we get a supersymmetric configuration of the CFT. Taking into account all $N=n_{1} n_{5}$ copies of the CFT we find that the states will have dimensions and charges

$$
\begin{aligned}
h_{\text {total }} & =\frac{1}{4}(2 n+1)^{2} n_{1} n_{5} \\
j_{\text {total }} & =-\frac{1}{2}(2 n+1) n_{1} n_{5} \\
\bar{h}_{\text {total }} & =\frac{1}{4} n_{1} n_{5} \\
\bar{j}_{\text {total }} & =-\frac{1}{2} n_{1} n_{5}
\end{aligned}
$$

In particular we have 'momentum' along the $S^{1}$, with

$$
h-\bar{h}=n(n+1) n_{1} n_{5}
$$

\subsection{Explicit representation of the states}

Let us construct explicitly the above CFT states. Consider one copy of the $c=6 \mathrm{CFT}$, in the $\mathrm{R}$ sector. The fermions have modes $\psi_{m}^{i}$. The 4 real fermions can be grouped into 2 complex fermions $\psi^{+}, \psi^{-}$which form a representation of $s u(2)$. $\left(\psi^{+}\right.$has $j=\frac{1}{2}$ and $\psi^{-}$ has $j=-\frac{1}{2}$.) The anti-commutation relations are

$$
\left\{\left(\psi^{+}\right)_{m}^{*}, \psi_{p}^{+}\right\}=\delta_{m+p, 0}, \quad\left\{\left(\psi^{-}\right)_{m}^{*}, \psi_{p}^{-}\right\}=\delta_{m+p, 0}
$$


The $s u(2)$ currents are

$$
J_{m}^{+}=\left(\psi^{-}\right)_{m-p}^{*} \psi_{p}^{+}, \quad J_{m}^{-}=\left(\psi^{+}\right)_{m-p}^{*} \psi_{p}^{-}, \quad J_{m}^{3}=\frac{1}{2}\left[\left(\psi^{-}\right)_{m-p}^{*} \psi_{p}^{-}-\left(\psi^{+}\right)_{m-p}^{*} \psi_{p}^{+}\right]
$$

The R ground state for $n=1$ in (2.4) has $j=-\frac{1}{2}$; we call this state $|1\rangle$. We can get the R ground state with $n=-1$ (which has $j=\frac{1}{2}$ ) by applying the zero modes $\psi_{0}^{+},\left(\psi^{-}\right)_{0}^{*}$, which is equivalent to applying $J_{0}^{+}$

$$
|-1\rangle=\left(\psi^{-}\right)_{0}^{*} \psi_{0}^{+}|1\rangle=J_{0}^{+}|1\rangle
$$

We see that we cannot apply $J_{-1}^{+}$to $|-1\rangle$, but we can apply $J_{-2}^{+}$getting the state with $n=-2$

$$
|-2\rangle=J_{-2}^{+} J_{0}^{+}|1\rangle=\left(\psi^{-}\right)_{-1}^{*} \psi_{-1}^{+}\left(\psi^{-}\right)_{0}^{*} \psi_{0}^{+}|1\rangle
$$

and so on. Returning to the full theory with $n_{1} n_{5}$ copies of the $c=6$ CFT we find that the currents are the sum of the currents in the individual copies

$$
J_{n}^{a, t o t a l}=\left(J_{n}^{a}\right)_{1}+\ldots\left(J_{n}^{a}\right)_{n_{1} n_{5}}
$$

and for $k \geq 0$

$$
|-k\rangle^{\text {total }}=\left(J_{-(2 k-2)}^{+, \text {total }}\right)^{n_{1} n_{5}}\left(J_{-(2 k-4)}^{+, \text {total }}\right)^{n_{1} n_{5}} \ldots\left(J_{-2}^{+, \text {total }}\right)^{n_{1} n_{5}}\left(J_{0}^{+, \text {total }}\right)^{n_{1} n_{5}}|1\rangle^{\text {total }}
$$

Similarly, for $k>1$

$$
|k\rangle^{\text {total }}=\left(J_{-(2 k-2)}^{-, \text {total }}\right)^{n_{1} n_{5}}\left(J_{-(2 k-4)}^{-, \text {total }}\right)^{n_{1} n_{5}} \ldots\left(J_{-2}^{-, \text {total }}\right)^{n_{1} n_{5}}|1\rangle^{\text {total }}
$$

\section{Constructing the gravity duals}

In [3] the 2-charge D1-D5 solutions were found by dualizing to the FP system, which has a fundamental string $(\mathrm{F})$ wrapped on $S^{1}$ carrying momentum (P) along $S^{1}$. Metrics for the vibrating string were constructed, and dualized back to get D1-D5 geometries. The general geometry was thus parametrized by the vibration profile $\vec{F}(v)$ of the $\mathrm{F}$ string. But a 1-parameter subfamily of these D1-D5 geometries had been found earlier [14, 15], by looking at extremal limits of the general axially symmetric D1-D5 geometry found in 16 .

We do not have an analogue of the procedure of [3] for 3-charge systems. We will follow instead the analogue of [14, 15] and take an extremal limit of the general 3-charge solution to obtain solutions with D1, D5 and P charges. Taking the limit needs some care, and it will be important to know in advance the properties of the CFT states for which we will be finding the duals. The procedure will give us the duals of the states $|n\rangle^{\text {total }}$ which were discussed in the last section. We will find that the dual geometries are completely smooth, with no horizon and no singularity. 


\subsection{Spectral flow in the gravity description}

In [14, 15] the following 2-charge D1-D5 solution was found (setting $Q_{1}=Q_{5}=Q$ for simplicity)

$$
\begin{aligned}
d s^{2} & =-\frac{1}{h}\left(d t^{2}-d y^{2}\right)+h f\left(d \theta^{2}+\frac{d r^{2}}{r^{2}+a^{2}}\right)-\frac{2 a Q}{h f}\left(\cos ^{2} \theta d y d \psi+\sin ^{2} \theta d t d \phi\right) \\
& +h\left[\left(r^{2}+\frac{a^{2} Q^{2} \cos ^{2} \theta}{h^{2} f^{2}}\right) \cos ^{2} \theta d \psi^{2}+\left(r^{2}+a^{2}-\frac{a^{2} Q^{2} \sin ^{2} \theta}{h^{2} f^{2}}\right) \sin ^{2} \theta d \phi^{2}\right]+d z_{i} d z_{i}
\end{aligned}
$$

where

$$
a=\frac{Q}{R}, \quad f=r^{2}+a^{2} \cos ^{2} \theta, \quad h=1+\frac{Q}{f}
$$

Let $R>>\sqrt{Q}$. In the region $r<<\sqrt{Q}$ the geometry (3.1) becomes

$$
\begin{aligned}
d s^{2}=- & \frac{\left(r^{2}+a^{2} \cos ^{2} \theta\right)}{Q}\left(d t^{2}-d y^{2}\right)+Q\left(d \theta^{2}+\frac{d r^{2}}{r^{2}+a^{2}}\right) \\
& -2 a\left(\cos ^{2} \theta d y d \psi+\sin ^{2} \theta d t d \phi\right)+Q\left(\cos ^{2} \theta d \psi^{2}+\sin ^{2} \theta d \phi^{2}\right)
\end{aligned}
$$

The change of coordinates

$$
\psi_{N S}=\psi-\tilde{\alpha} \frac{a}{Q} y+(\tilde{\alpha}-1) \frac{a}{Q} t, \quad \phi_{N S}=\phi+(\tilde{\alpha}-1) \frac{a}{Q} y-\tilde{\alpha} \frac{a}{Q} t
$$

with $\tilde{\alpha}=1$ brings (3.3) to the form $A d S_{3} \times S^{3}$

$$
d s^{2}=-\frac{\left(r^{2}+a^{2}\right)}{Q} d t^{2}+\frac{r^{2}}{Q} d y^{2}+Q \frac{d r^{2}}{r^{2}+a^{2}}+Q\left(d \theta^{2}+\cos ^{2} \theta d \psi_{N S}^{2}+\sin ^{2} \theta d \phi_{N S}^{2}\right)
$$

The solution (3.1) describes the asymptotically flat solution created by D1-D5 branes, with fermions periodic around the $y$ circle. The 'near-region' part (3.3) describes the gravity dual of the appropriate CFT state, in the $\mathrm{R}$ sector. The change of coordinates (3.4) gives 'spectral flow' in the gravity description, and with $\tilde{\alpha}=1$ we reach (3.5) which gives the gravity dual of the NS sector version of the CFT state [14, 15].

The 3-charge D1-D5-P states that we wish to describe are obtained by further spectral flow applied to the state described by (3.3). From (3.4) we see that the 'near region' geometry dual to these states is just $A d S_{3} \times S^{3}$ described in new coordinates. (The state $|n\rangle^{\text {total }}$ is generated by $\tilde{\alpha}=-n$.) Thus we may anticipate a 'capped' geometry for these states. It is not obvious though that the near region solution can be continued to flat space at infinity, while keeping the solution BPS as well as smooth (the analogue of (3.1)), but we will see that in fact we do get such a solution. 


\subsection{The nonextremal 3-charge solution, with angular momen- tum}

The nonextremal 3-charge metric with rotation was given in [16, but we will also need the 2-form gauge field (which was not listed in [16]) so we derive this solution in the Appendix. We follow a different method however from [16]; we take a neutral rotating hole, and by a sequence of boosts and dualities, add the three charges. We find

$$
\begin{aligned}
d s^{2}= & -\left(1-\frac{M \cosh ^{2} \delta_{p}}{f}\right) \frac{d t^{2}}{\sqrt{H_{1} H_{5}}}+\left(1+\frac{M \sinh ^{2} \delta_{p}}{f}\right) \frac{d y^{2}}{\sqrt{H_{1} H_{5}}}-\frac{M \sinh 2 \delta_{p}}{f \sqrt{H_{1} H_{5}}} d t d y \\
+ & f \sqrt{H_{1} H_{5}}\left(\frac{r^{2} d r^{2}}{\left(r^{2}+a_{1}^{2}\right)\left(r^{2}+a_{2}^{2}\right)-M r^{2}}+d \theta^{2}\right) \\
+ & {\left[\left(r^{2}+a_{1}^{2}\right) \sqrt{H_{1} H_{5}}+\frac{\left(a_{2}^{2}-a_{1}^{2}\right) K_{1} K_{5} \cos ^{2} \theta}{\sqrt{H_{1} H_{5}}}\right] \cos ^{2} \theta d \psi^{2} } \\
+ & {\left[\left(r^{2}+a_{2}^{2}\right) \sqrt{H_{1} H_{5}}+\frac{\left(a_{1}^{2}-a_{2}^{2}\right) K_{1} K_{5} \sin ^{2} \theta}{\sqrt{H_{1} H_{5}}}\right] \sin ^{2} \theta d \phi^{2} } \\
+ & \frac{M}{f \sqrt{H_{1} H_{5}}}\left(a_{1} \cos ^{2} \theta d \psi+a_{2} \sin ^{2} \theta d \phi\right)^{2} \\
+ & \frac{2 M \cos { }^{2} \theta}{f \sqrt{H_{1} H_{5}}}\left[\left(a_{1} \cosh \delta_{1} \cosh \delta_{5} \cosh \delta_{p}-a_{2} \sinh \delta_{1} \sinh \delta_{5} \sinh \delta_{p}\right) d t\right. \\
& \left.+\left(a_{2} \sinh \delta_{1} \sinh \delta_{5} \cosh \delta_{p}-a_{1} \cosh \delta_{1} \cosh \delta_{5} \sinh \delta_{p}\right) d y\right] d \psi \\
+ & \frac{2 M \sin { }^{2} \theta}{f \sqrt{H_{1} H_{5}}}\left[\left(a_{2} \cosh \delta_{1} \cosh \delta_{5} \cosh \delta_{p}-a_{1} \sinh \delta_{1} \sinh \delta_{5} \sinh \delta_{p}\right) d t\right. \\
& \left.+\left(a_{1} \sinh \delta_{1} \sinh \delta_{5} \cosh \delta_{p}-a_{2} \cosh \delta_{1} \cosh \delta_{5} \sinh \delta_{p}\right) d y\right] d \phi+\sqrt{\frac{H_{1}}{H_{5}} \sum_{i=1}^{4} d z_{i}^{2}} \\
C_{2} & \frac{M \cos { }^{2} \theta}{f H_{1}}\left[\left(a_{2} \cosh \delta_{1} \sinh \delta_{5} \cosh \delta_{p}-a_{1} \sinh \delta_{1} \cosh \delta_{5} \sinh \delta_{p}\right) d t\right. \\
& \left.+\left(a_{1} \sinh \delta_{1} \cosh \delta_{5} \cosh \delta_{p}-a_{2} \cosh \delta_{1} \sinh \delta_{5} \sinh \delta_{p}\right) d y\right] \wedge d \psi \\
+ & \frac{M \sin { }^{2} \theta}{f H_{1}}\left[\left(a_{1} \cosh \delta_{1} \sinh \delta_{5} \cosh \delta_{p}-a_{2} \sinh \delta_{1} \cosh \delta_{5} \sinh \delta_{p}\right) d t\right. \\
& \left.+\left(a_{2} \sinh \delta_{1} \cosh \delta_{5} \cosh \delta_{p}-a_{1} \cosh \delta_{1} \sinh \delta_{5} \sinh \delta_{p}\right) d y\right] \wedge d \phi \\
- & \frac{M \sinh 2 \delta_{1}}{2 f H_{1}} d t \wedge d y-\frac{M \sinh 2 \delta_{5}}{2 f H_{1}}\left(r^{2}+a_{2}^{2}+M \sinh \delta_{1}\right) \cos { }^{2} \theta d \psi \wedge d \phi \\
= & \frac{H_{1}}{H_{5}} \\
e^{2 \Phi} & \\
& \\
&
\end{aligned}
$$


Here

$$
\begin{gathered}
f=r^{2}+a_{1}^{2} \sin ^{2} \theta+a_{2}^{2} \cos ^{2} \theta \\
H_{i} \equiv 1+K_{i}=1+\frac{M \sinh ^{2} \delta_{i}}{f}, \quad i=1,5
\end{gathered}
$$

\section{The Extremal Limit}

\subsection{Conserved charges}

We want to take an extremal limit of the above solution. We take this limit while keeping the conserved charges fixed to the values that describe the states $|n\rangle^{\text {total }}$ defined in section 2. Thus the solution should describe $n_{1}$ D1 branes, $n_{5}$ D5 branes, $n_{p}=n(n+1) n_{1} n_{5}$ units of momentum, and angular momenta

$$
J_{\psi}=-\bar{j}+j=-n n_{1} n_{5}, \quad J_{\phi}=-\bar{j}-j=(n+1) n_{1} n_{5}
$$

The volume of the $T^{4}$ is $V$ and the length of the $S^{1}$ is $2 \pi R$. The 10-D Newton's constant is $G^{(10)}=8 \pi^{6} g^{2} \alpha^{4}$. If we dimensionally reduce along $T^{4}, S^{1}$ then we get the 5 -D Newton's constant

$$
G^{(5)}=\frac{G^{(10)}}{V(2 \pi R)}
$$

From the solution given above we get

$$
\begin{aligned}
Q_{1} & \equiv \frac{M}{2} \sinh 2 \delta_{1}=\frac{(2 \pi)^{4} g \alpha^{\prime 3}}{V} n_{1} \\
Q_{5} & \equiv \frac{M}{2} \sinh 2 \delta_{p}=g \alpha^{\prime} n_{5} \\
Q_{p} & \equiv \frac{M}{2} \sinh 2 \delta_{p}=\frac{(2 \pi)^{4} g^{2} \alpha^{\prime 4}}{V R^{2}} n_{p}=\frac{(2 \pi)^{4} g^{2} \alpha^{\prime 4}}{V R^{2}} n(n+1) n_{1} n_{5}
\end{aligned}
$$

It will be useful to define the length scale

$$
a=\frac{\sqrt{Q_{1} Q_{5}}}{R}
$$

We observe that

$$
Q_{p}=n(n+1) a^{2}
$$

The angular momenta are

$$
\begin{aligned}
& J_{\psi}=-M\left(a_{1} \cosh \delta_{1} \cosh \delta_{5} \cosh \delta_{p}-a_{2} \sinh \delta_{1} \sinh \delta_{5} \sinh \delta_{p}\right) \frac{\pi}{4 G^{(5)}}=-n n_{1} n_{5} \\
& J_{\phi}=-M\left(a_{2} \cosh \delta_{1} \cosh \delta_{5} \cosh \delta_{p}-a_{1} \sinh \delta_{1} \sinh \delta_{5} \sinh \delta_{p}\right) \frac{\pi}{4 G^{(5)}}=(n+1) n_{1} n_{5}
\end{aligned}
$$


It will be helpful to define

$$
\begin{aligned}
& -\frac{M}{\sqrt{Q_{1} Q_{5}}}\left(a_{1} \cosh \delta_{1} \cosh \delta_{5} \cosh \delta_{p}-a_{2} \sinh \delta_{1} \sinh \delta_{5} \sinh \delta_{p}\right) \equiv \gamma_{1} \\
& -\frac{M}{\sqrt{Q_{1} Q_{5}}}\left(a_{2} \cosh \delta_{1} \cosh \delta_{5} \cosh \delta_{p}-a_{1} \sinh \delta_{1} \sinh \delta_{5} \sinh \delta_{p}\right) \equiv \gamma_{2}
\end{aligned}
$$

Then (4.9) implies

$$
\gamma_{1}=-a n, \quad \gamma_{2}=a(n+1)
$$

\subsection{Taking the extremal limit}

To get the extremal limit we must take

$$
M \rightarrow 0, \quad \delta_{i} \rightarrow \infty \quad(i=1,5, p)
$$

keeping the $Q_{i}$ fixed. This gives

$$
\begin{aligned}
\cosh ^{2} \delta_{i} & =\frac{Q_{i}}{M}+\frac{1}{2}+O(M) \\
\sinh ^{2} \delta_{i} & =\frac{Q_{i}}{M}-\frac{1}{2}+O(M)
\end{aligned}
$$

We must also take suitable limits of $a_{1}, a_{2}$ so that the angular momenta are held fixed. It is useful to invert (4.9):

$$
\begin{aligned}
& a_{1}=-\frac{\sqrt{Q_{1} Q_{5}}}{M} \frac{\gamma_{1} \cosh \delta_{1} \cosh \delta_{5} \cosh \delta_{p}+\gamma_{2} \sinh \delta_{1} \sinh \delta_{5} \sinh \delta_{p}}{\cosh ^{2} \delta_{1} \cosh ^{2} \delta_{5} \cosh ^{2} \delta_{p}-\sinh ^{2} \delta_{1} \sinh ^{2} \delta_{5} \sinh ^{2} \delta_{p}} \\
& a_{2}=-\frac{\sqrt{Q_{1} Q_{5}}}{M} \frac{\gamma_{2} \cosh \delta_{1} \cosh \delta_{5} \cosh \delta_{p}+\gamma_{1} \sinh \delta_{1} \sinh \delta_{5} \sinh \delta_{p}}{\cosh ^{2} \delta_{1} \cosh ^{2} \delta_{5} \cosh ^{2} \delta_{p}-\sinh ^{2} \delta_{1} \sinh ^{2} \delta_{5} \sinh ^{2} \delta_{p}}
\end{aligned}
$$

Using (4.12) we find

$$
\begin{aligned}
a_{1} & =-\left(\gamma_{1}+\gamma_{2}\right) \eta \sqrt{\frac{Q_{p}}{M}}-\frac{\gamma_{1}-\gamma_{2}}{4} \sqrt{\frac{M}{Q_{p}}}+O\left(M^{3 / 2}\right) \\
& =-a \eta \sqrt{\frac{Q_{p}}{M}}+a \frac{2 n+1}{4} \sqrt{\frac{M}{Q_{p}}}+O\left(M^{3 / 2}\right) \\
a_{2} & =-\left(\gamma_{1}+\gamma_{2}\right) \eta \sqrt{\frac{Q_{p}}{M}}+\frac{\gamma_{1}-\gamma_{2}}{4} \sqrt{\frac{M}{Q_{p}}}+O\left(M^{3 / 2}\right) \\
& =-a \eta \sqrt{\frac{Q_{p}}{M}}-a \frac{2 n+1}{4} \sqrt{\frac{M}{Q_{p}}}+O\left(M^{3 / 2}\right)
\end{aligned}
$$


where we have defined the dimensionless combination

$$
\eta \equiv \frac{Q_{1} Q_{5}}{Q_{1} Q_{5}+Q_{1} Q_{p}+Q_{5} Q_{p}}
$$

and in the second equalities we have used the specific values for $\gamma_{1}$ and $\gamma_{2}$ given in (4.10).

We thus see that for generic values of $\gamma_{1}, \gamma_{2}$ and $Q_{p}$ the parameters $a_{1}$ and $a_{2}$ diverge when $M \rightarrow 0$. There are two exceptions:

(a) $Q_{p}=0$, which is the case considered in [14, 15]; in this case $a_{1}$ and $a_{2}$ go to finite values when $M \rightarrow 0$.

(b) $\bar{j} \sim\left(\gamma_{1}+\gamma_{2}\right) / 2=0$; in this case $a_{1}$ and $a_{2}$ go to zero as $\sqrt{M}$ in the extremal limit. This case was studied in [16, 17.

In the case of interest to us both $Q_{p}$ and $\gamma_{1}+\gamma_{2}$ are non-zero and thus $a_{1}$ and $a_{2}$ have divergent limits when $M \rightarrow 0$. This might seem to pose a problem for the finiteness of the metric in the extremal limit. Note however that $a_{1}$ and $a_{2}$ have the same divergent part, so one of the expressions occurring in the metric

$$
a_{1}^{2}-a_{2}^{2} \rightarrow-a^{2} \eta(2 n+1)
$$

is seen to be finite. Note that we need to keep terms up to $O(\sqrt{M})$ in the expansion of $a_{1}, a_{2}$ in obtaining the above limit; higher order terms can however be discarded. Similar care has to be taken in other computations below.

We also encounter the expressions $r^{2}+a_{i}^{2}$. Define a new radial coordinate

$$
r_{N}^{2}=r^{2}+\frac{a^{2} \eta^{2} Q_{p}}{M}-\beta^{2}
$$

The term which diverges when $M$ goes to zero has been chosen to cancel the divergence of $a_{1}^{2}$ and $a_{2}^{2}$, and $\beta$ is a finite constant that we will fix later. Note that $r^{2}$ goes to $-\infty$ down the throat in the extremal limit but $r_{N}^{2}$ will reach a finite value. We find the following as $M \rightarrow 0$ :

$$
\begin{aligned}
r^{2}+a_{1}^{2} & =r_{N}^{2}+\beta^{2}-a^{2} \eta \frac{2 n+1}{2} \\
r^{2}+a_{2}^{2} & =r_{N}^{2}+\beta^{2}+a^{2} \eta \frac{2 n+1}{2} \\
f & =r^{2}+a_{1}^{2} \sin ^{2} \theta+a_{2}^{2} \cos ^{2} \theta \\
& =r_{N}^{2}+\left(\beta^{2}-a^{2} \eta \frac{2 n+1}{2}\right) \sin ^{2} \theta+\left(\beta^{2}+a^{2} \eta \frac{2 n+1}{2}\right) \cos ^{2} \theta
\end{aligned}
$$

The coefficient of $d r^{2}$ has the factor $\frac{r^{2}}{\left(r^{2}+a_{1}^{2}\right)\left(r^{2}+a_{2}^{2}\right)-M r^{2}}$. If we choose

$$
\beta^{2}=\frac{\eta a^{2}}{2}
$$


we are left with only a quadratic in the denominator. To see this note that the denominator is

$$
\begin{aligned}
\left(r^{2}+\right. & \left.a_{1}^{2}\right)\left(r^{2}+a_{2}^{2}\right)-M r^{2} \\
& =\left(r_{N}^{2}+\beta^{2}-a^{2} \eta \frac{2 n+1}{2}\right)\left(r_{N}^{2}+\beta^{2}+a^{2} \eta \frac{2 n+1}{2}\right)-M\left(r_{N}^{2}-\frac{a^{2} \eta^{2} Q_{p}}{M}+\beta^{2}\right) \\
& \rightarrow\left(r_{N}^{2}+\beta^{2}-a^{2} \eta \frac{2 n+1}{2}\right)\left(r_{N}^{2}+\beta^{2}+a^{2} \eta \frac{2 n+1}{2}\right)+a^{2} \eta^{2} Q_{p} \\
& =r_{N}^{2}\left(r_{N}^{2}+\eta a^{2}\right)
\end{aligned}
$$

The numerator is $r^{2} d r^{2}=r_{N}^{2} d r_{N}^{2}$, and we get a cancellation of the factors $r_{N}^{2}$. We will see below that in the extremal metric the point $r_{N}=0$ acts like an origin of polar coordinates, so the choice (4.19) is the correct one to define a coordinate $r_{N}$ with range $(0, \infty)$.

We also find that other terms in the metric and gauge field are finite in the extremal limit; this can be verified using (4.12),(4.14). We get the extremal solution (in the string frame)

$$
\begin{aligned}
d s^{2} & =-\frac{1}{h}\left(d t^{2}-d y^{2}\right)+\frac{Q_{p}}{h f}(d t-d y)^{2}+h f\left(\frac{d r_{N}^{2}}{r_{N}^{2}+a^{2} \eta}+d \theta^{2}\right) \\
& +h\left(r_{N}^{2}-n a^{2} \eta+\frac{(2 n+1) a^{2} \eta Q_{1} Q_{5} \cos ^{2} \theta}{h^{2} f^{2}}\right) \cos ^{2} \theta d \psi^{2} \\
& +h\left(r_{N}^{2}+(n+1) a^{2} \eta-\frac{(2 n+1) a^{2} \eta Q_{1} Q_{5} \sin ^{2} \theta}{h^{2} f^{2}}\right) \sin ^{2} \theta d \phi^{2} \\
& +\frac{a^{2} \eta^{2} Q_{p}}{h f}\left(\cos ^{2} \theta d \psi+\sin ^{2} \theta d \phi\right)^{2} \\
& +\frac{2 a \sqrt{Q_{1} Q_{5}}}{h f}\left[n \cos ^{2} \theta d \psi-(n+1) \sin ^{2} \theta d \phi\right](d t-d y) \\
& -\frac{2 a \eta \sqrt{Q_{1} Q_{5}}}{h f}\left[\cos ^{2} \theta d \psi+\sin ^{2} \theta d \phi\right] d y+\sqrt{\frac{H_{1}}{H_{5}}} \sum_{i=1}^{4} d z_{i}^{2} \\
C_{2} & =\frac{a \sqrt{Q_{1} Q_{5}} \cos ^{2} \theta}{H_{1} f}(-(n+1) d t+n d y) \wedge d \psi \\
& +\frac{a \sqrt{Q_{1} Q_{5}} \sin ^{2} \theta}{H_{1} f}(n d t-(n+1) d y) \wedge d \phi \\
& +\frac{a \eta Q_{p}}{\sqrt{Q_{1} Q_{5}} H_{1} f}\left(Q_{1} d t+Q_{5} d y\right) \wedge\left(\cos ^{2} \theta d \psi+\sin ^{2} \theta d \phi\right) \\
& -\frac{Q_{1}}{H_{1} f} d t \wedge d y-\frac{Q_{5} \cos ^{2} \theta}{H_{1} f}\left(r_{N}^{2}+(n+1) a^{2} \eta+Q_{1}\right) d \psi \wedge d \phi \\
e^{2 \Phi} & \frac{H_{1}}{H_{5}}(n)
\end{aligned}
$$




$$
\begin{aligned}
& f=r_{N}^{2}-a^{2} \eta n \sin ^{2} \theta+a^{2} \eta(n+1) \cos ^{2} \theta \\
& h=\sqrt{H_{1} H_{5}}, H_{1}=1+\frac{Q_{1}}{f}, H_{5}=1+\frac{Q_{5}}{f}
\end{aligned}
$$

\section{Regularity of the Solution}

\subsection{Regularity of the metric}

A sufficient condition for the metric to be regular is that the coefficients of both the metric and the inverse metric be twice differentiable functions of the coordinates. In turn, the inverse metric is well-defined if the metric is smooth and the determinant is non-vanishing. For the metric (4.21)

$$
\sqrt{-g}=\frac{H_{1}}{H_{5}} h f r_{N} \sin \theta \cos \theta
$$

The function $f$ does vanish on a hypersurface, but the combination $h f$ takes a finite value when $f \rightarrow 0$

$$
f \rightarrow 0 \Rightarrow h f \rightarrow \sqrt{Q_{1} Q_{5}}
$$

The function $f$ appears explicitly in the metric only in the combination $h f$; thus we do not get singularities at $f \rightarrow 0$.

A little algebra shows that

$$
Q_{1}+f>0, \quad Q_{5}+f>0
$$

everywhere. (To check this one needs to note that $n$ is an integer; for fractional $n$ the above expressions can become negative at some points.) Thus the factor $\frac{H_{1}}{H_{5}}$ in (5.1) is regular and nowhere vanishing.

We have factors of $h$ in the second and third lines of (4.21). Since

$$
h=\frac{\sqrt{\left(Q_{1}+f\right)\left(Q_{5}+f\right)}}{f}
$$

we have $h \rightarrow \infty$ as $f \rightarrow 0$. But a little algebra shows that when $f \rightarrow 0$ the brackets multiplying $h$ reduce to $f$ in each case, so we again get the finite combination $h f$.

The vanishing of $\sqrt{-g}$ for $\theta=0$ and $\pi / 2$ does not correspond to a singularity of the metric but only signals the degeneration of polar coordinates at the north and south pole of $S^{3}$.

The most nontrivial limit arises at $r_{N} \rightarrow 0$. Let

$$
\phi \rightarrow \tilde{\phi}=\phi+\frac{a n}{\sqrt{Q_{1} Q_{5}}} y \quad \psi \rightarrow \tilde{\psi}=\psi-\frac{a(n+1)}{\sqrt{Q_{1} Q_{5}}} y
$$


In these coordinates, the metric expanded around $r_{N} \rightarrow 0$ has the following form:

$$
\begin{aligned}
d s^{2}= & \frac{h f}{\eta a^{2}}\left(d r_{N}^{2}+\frac{a^{2}}{Q_{1} Q_{5}} r_{N}^{2} d y^{2}\right) \\
& +h f\left(d \theta^{2}+\tilde{g}_{\psi \psi} d \tilde{\psi}^{2}+\tilde{g}_{\phi \phi} d \tilde{\phi}^{2}+2 \tilde{g}_{\psi \phi} d \tilde{\psi} d \tilde{\phi}\right) \\
& +g_{t t} d t^{2}+2 g_{t \psi} d t d \tilde{\psi}+2 g_{t \phi} d t d \tilde{\phi}+d s_{T^{4}}^{2}
\end{aligned}
$$

where now

$$
f=\eta a^{2}(n+1) \cos ^{2} \theta-\eta a^{2} n \sin ^{2} \theta
$$

The coefficients $\tilde{g}$ and $g$ in the equation above are differentiable functions of $r_{N}$ and $\theta$ that do not vanish as $r_{N} \rightarrow 0$ for generic values of $\theta$; their explicit form is not important for our argument.

There are several points to note about the above expansion:

(a) Before the coordinate transformation (5.5) we had the identifications $(y, \psi, \phi) \sim$ $\left(y+2 \pi m_{3} R, \psi+2 \pi m_{1}, \phi+2 \pi m_{2}\right)$. Recalling that $R=\frac{\sqrt{Q_{1} Q_{5}}}{a}$ we see that the transformation (5.5) gives new variables that have a similar identification

$$
(y, \tilde{\psi}, \tilde{\phi}) \sim\left(y+2 \pi m_{3}^{\prime} R, \tilde{\psi}+2 \pi m_{1}^{\prime}, \tilde{\phi}+2 \pi m_{2}^{\prime}\right)
$$

(b) Noting that $y$ is periodic with period $2 \pi R$ and recalling that $R=\frac{\sqrt{Q_{1} Q_{5}}}{a}$ we see that the coefficient of $d y^{2}$ is just right to make $r_{N}, y$ polar coordinates at the origin in the $r_{N}, y$ space

$$
\frac{h f}{\eta a^{2}}\left(d r_{N}^{2}+\frac{a^{2}}{Q_{1} Q_{5}} r_{N}^{2} d y^{2}\right)=\frac{h f}{\eta a^{2}}\left(d r_{N}^{2}+\frac{r_{N}^{2}}{R^{2}} d y^{2}\right)
$$

and thus there is no conical defect singularity at $r_{N}=0$.

(c) There is no term $d t d y$ at leading order in $r_{N}$; this cross term is absorbed entirely in the terms $d \tilde{\psi} d y, d \tilde{\phi} d y$. Note that if we did have a residual term $d t d y$ we would not get smoothness where the $y$ circle shrinks. Indeed, to reach local orthogonal coordinates we would need to define new coordinates

$$
y^{\prime}=y, \quad t^{\prime}=t+c y, \quad c \neq 0
$$

The identification vector in the $(y, t)$ space is $(y, t) \sim(y+2 \pi R, t)$, which implies the identification

$$
\left(y^{\prime}, t^{\prime}\right) \sim\left(y^{\prime}+2 \pi R, t^{\prime}+2 \pi c R\right)
$$

Such an identification gives a singularity at $r_{N}=0$ where the $y$ circle shrinks, and also generates closed timelike curves if $t^{\prime}$ is a timelike direction. Since we get $c=0$, we avoid both of these potential problems.

Nothing special happens at $r_{N}=0, \theta=0, \pi / 2$; we just have the degeneration of spherical coordinates at the poles. We conclude that the metric is regular everywhere. 


\subsection{Absence of closed timelike curves}

In the past, geometries have been constructed with D1-D5-P charges and angular momentum, and it was found that for high enough angular momentum the geometries had closed timelike curves [18, 19, 20]. Our geometries on the other hand are conjectured to be dual to actual microstates of the CFT, so we expect them to be free of pathologies.

There are three periodic variables in our geometry $-y, \psi, \phi$. Following the path

$$
(\delta y, \delta \psi, \delta \phi)=\left(n_{1} R \epsilon, n_{2} \epsilon, n_{3} \epsilon\right)
$$

gives a closed curve for any integers $n_{i}$. This curve is spacelike at large $r_{N}$, but a curve like this gave a closed timelike curve at small $r_{N}$ in the metric studied in [19. We thus ask if the vector (5.12) can become timelike anywhere; if it does become timelike for some $r_{N}, \theta$ then we get a closed timelike curve. If the vector (15.12) becomes timelike for nonintegral $n_{i}$ then we get a timelike path that passes arbitrarily close to itself, which is pathological too; thus we investigate if the vector

$$
V^{y}=\alpha, \quad V^{\psi}=\beta, \quad V^{\phi}=\gamma
$$

can become timelike anywhere. Note that adding components $V^{r}, V^{\theta}$ makes $V$ more spacelike, while having a part $V^{t} \neq 0$ makes the path non-closed.

The norms of the vectors (15.13) are given by the metric $g_{a b}$ restricted to $a, b=$ $y, \psi, \phi$. Let $\tilde{g}$ be the determinant of the metric restricted to these three coordinates. The components $g_{a b}$ of the restricted metric are regular and $\tilde{g}$ is positive at infinity. If some direction in the 3-torus spanned by $y, \psi, \phi$ is to become timelike then we must have a vanishing of $\tilde{g}$ somewhere. But an explicit evaluation of this determinant gives

$$
\tilde{g}=\frac{r_{N}^{2} \sin ^{2} \theta \cos ^{2} \theta}{\sqrt{\left(Q_{1}+f\right)\left(Q_{5}+f\right)}}\left[\left(r_{N}^{2}+\eta a^{2}\right)\left(f+Q_{1}+Q_{5}+a^{2} n(n+1)\right)+\frac{Q_{1} Q_{5}}{\eta}\right]
$$

The fact that the combinations $Q_{1}+f$ and $Q_{5}+f$ are always positive ensures that the determinant above only vanishes at $r_{N}=0$ or $\theta=0$ or $\pi / 2$. Our explicit analysis of the metric in the regions around $r_{N}=0$ and $\theta=0$ or $\pi / 2$ shows that no closed time-like curves appear even at these points; the vanishing of $\tilde{g}$ just signals the vanishing of one of the three periodic coordinates.

\subsection{Regularity of $\Phi, C_{A B}$}

The dilaton given by (4.23) can be written as

$$
e^{2 \Phi}=\frac{Q_{1}+f}{Q_{5}+f}
$$

From (5.3) we see that $\Phi$ is regular everywhere. 
Let us finally check that the RR field is not singular. Since its coefficients are differentiable functions of $r_{N}$ and $\theta$ for all values of the coordinates, singularities can come only from places where the coordinate system degenerates, i.e. at $\theta=0$ and $\pi / 2$ (nothing special happens at $r_{N}=0$ ).

At $\theta=\pi / 2$ we see that the components of $C$ are regular. At $\theta=0$, where the $\phi$ coordinate becomes ill defined, the only component that is potentially singular is $C_{\phi \psi}$ :

$$
\begin{aligned}
C_{\phi \psi} & =\frac{Q_{5}}{f+Q_{1}}\left(r_{N}^{2}+(n+1) \eta a^{2}+Q_{1}\right) \cos ^{2} \theta \\
& =Q_{5} \cos ^{2} \theta+\frac{Q_{5} a^{2}}{f+Q_{1}}(1+2 n) \sin ^{2} \theta \cos ^{2} \theta \\
& \approx Q_{5}\left(1-\theta^{2}\right)+\frac{Q_{5} a^{2}}{f+Q_{1}}(1+2 n) \theta^{2}=Q_{5}+O\left(\theta^{2}\right)
\end{aligned}
$$

The leading term can be removed by a gauge transformation:

$$
C_{\phi \psi} \rightarrow C_{\phi \psi}-Q_{5}
$$

and $C_{\phi \psi}$ becomes regular. This is just the gauge transformation arising from a magnetic monopole potential. Such a potential is expected since the D5 branes produce a magnetic charge for $C_{2}$.

\subsection{Absence of a horizon}

Since the geometry is regular, singularity theorems suggest that there is no horizon. We can check the absence of a horizon explicitly in the following way. There is no horizon if from every point in the geometry we can find a path to asymptotic infinity such that the tangent along the path lies in the forward light cone everywhere.

To show that there is such a path, we will argue that at each point in the spacetime we can find a timelike vector in the forward light cone which has a nonzero positive $V^{r}$; thus following these vectors we can reach $r_{N} \rightarrow \infty$ along a timelike path. Suppose we can find a continuous timelike vector field $V$ with $V^{r}=0$. Since $d r_{N}$ appears in the metric only in the form $\frac{h f d r_{N}^{2}}{r_{N}^{2}+a^{2} \eta}$ we see that we can add a part $V^{r}>0$ to the vector at each point while still keeping it timelike. (If the vector field is continuous then it lies in the forward light cone everywhere if we choose it to lie in the forward light cone at infinity.)

We will find the vector field $V$ in the subspace spanned by the coordinates $t, y, \psi, \phi$. The determinant of the metric restricted to these four directions is

$$
\hat{g}=-r_{N}^{2}\left(r_{N}^{2}+\eta a^{2}\right) \sin ^{2} \theta \cos ^{2} \theta
$$

and is seen to be negative everywhere. Thus the tangent plane spanned by these directions has signature $(-1,1,1,1)$ everywhere. The region of interest is spanned by a single 
coordinate patch (apart from coordinate degeneration at the poles; this causes no difficulties for the argument). We can thus find a timelike direction in each tangent plane, which is continuous and in the forward light cone at $r_{N} \rightarrow \infty$.

As mentioned above, adding a sufficiently small $V^{r}$ keeps this vector field timelike and in the forward cone (it is important that the coefficient of $d r_{N}^{2}$ does not diverge anywhere, so the allowed $V^{r}$ can be bounded below). Following this vector field we reach $r_{N} \rightarrow \infty$, and thus there is no horizon.

\section{Comparison with the solution in [8]}

The metric in [8] was expressed somewhat implicitly, but with some algebraic manipulation it can be brought to the form

$$
\begin{aligned}
d s^{2}= & -\frac{d t^{2}-d y^{2}}{h}+\frac{\nu(\nu+1) \tilde{a}^{2}}{h f}(d t+d y)^{2}+h f\left(\frac{d r^{2}}{r^{2}+\tilde{a}^{2}}+d \theta^{2}\right) \\
& +h\left[r^{2}-\nu \tilde{a}^{2}+\frac{(2 \nu+1) \tilde{a}^{2} Q^{2} \cos ^{2} \theta}{h^{2} f^{2}}\right] \cos ^{2} \theta d \psi^{2} \\
& +h\left[r^{2}+(\nu+1) \tilde{a}^{2}-\frac{(2 \nu+1) \tilde{a}^{2} Q^{2} \sin ^{2} \theta}{h^{2} f^{2}}\right] \sin ^{2} \theta d \phi^{2} \\
& +\frac{\nu(\nu+1) \tilde{a}^{4}}{h f}\left(\cos ^{2} \theta d \psi-\sin ^{2} \theta d \phi\right)^{2} \\
& -\frac{2 \tilde{a} Q \cos ^{2} \theta}{h f}\left[\nu \frac{Q-(1+\nu) \tilde{a}^{2}}{Q} d t+(\nu+1) \frac{Q-\nu \tilde{a}^{2}}{Q} d y\right] d \psi \\
& -\frac{2 \tilde{a} Q \sin ^{2} \theta}{h f}\left[(\nu+1) \frac{Q+\nu \tilde{a}^{2}}{Q} d t+\nu \frac{Q+(\nu+1) \tilde{a}^{2}}{Q} d y\right] d \phi
\end{aligned}
$$

where

$$
\begin{aligned}
& f=r^{2}+\tilde{a}^{2}(\nu+1) \cos ^{2} \theta-\tilde{a}^{2} \nu \sin ^{2} \theta \\
& h=1+\frac{Q}{f}
\end{aligned}
$$

We have denoted by $\tilde{a}$ what in $[8]$ has been called $a$, to distinguish it from the analogous parameter appearing in our metric (4.21). Locally, the metric above reduces to ours (if we set $Q_{1}=Q_{5}=Q$ in our metric) after the following redefinitions of parameters and change of coordinates:

$$
\begin{aligned}
& \tilde{a}^{2}=a^{2} \eta, \quad \nu=n, \quad \psi \rightarrow-\psi, \quad r=r_{N} \\
& t \rightarrow t \cosh \delta-y \sinh \delta, \quad y \rightarrow-y \cosh \delta+t \sinh \delta
\end{aligned}
$$

with

$$
e^{-2 \delta}=\eta
$$


So essentially the two metrics are related by a boost in the $y$ direction. But since $y$ is a compact direction this boost is not a symmetry; identification of points is altered by the boost. It has been argued in [8] that the solution is smooth; it is not immediately obvious to us though how the identifications used there avoid shifts like (5.11) and the corresponding potential singularities. The charges of [8] appear to differ from the ones that we have, so the solutions there might correspond to a somewhat different class of 3 -charge states.

\section{Discussion}

We have constructed extremal D1-D5 -P solutions dual to a special subset of CFT states. The solutions were smooth, with no singularity or horizon. The solutions thus look like Fig.1(d) rather than Fig.1(c). These results support the general conjecture [7] that individual microstates of a black hole do not look like the naive picture of a black hole - 'empty space inside the horizon with a central singularity'. Rather, the horizon arises only as an effective construct when we coarse-graining over microstates. Some other interesting results obtained recently also throw light on the nontrivial size associated to high entropy states [21, 22, 23, 24, in many cases by representing them as supertubes 25. Topological field theories also offer hints of a 'blow up' of states in the gravity description [26].

The states we have looked at are a small subfamily of all the 3-charge states. They also are not very generic states since they carry a large angular momentum. One might therefore argue that low angular momentum states might still look like Fig.1(c). To throw some light on this issue let us recall the case of 2-charge states. The maximally rotating D1-D5 solutions were found first [14, 15, by taking limits of the general solutions in [16]. These solutions turned out to be 'capped' instead of extending to a pointlike singularity at $r=0$. The general D1-D5 solutions were found in [3]; the generic solution had no angular momentum but was still capped before reaching $r=0$. The reason can be seen best in the dual FP language. In the maximally rotating solution the $\mathrm{F}$ string carries the momentum $\mathrm{P}$ by swinging in a uniform helix, and the nonzero transverse size of the helix cuts off the geometry before $r=0$. But we can let half the $\mathrm{F}$ string swing clockwise and the other half anticlockwise; this still carries the same momentum $\mathrm{P}$ but gives no net angular momentum. Note however that we still get the nonzero transverse size, and hence the 'cap' to the throat. At a technical level, it was noted in [3] that angular momentum makes its presence felt in two kinds of terms: $\sim \operatorname{adtd\phi }$ and $\sim \frac{d r^{2}}{r^{2}+a^{2}}$. For states with no net rotation the former type of term cancels out (different parts of the geometry contribute with different signs to $a$ ) but the latter type of term survives since $a^{2}$ can only get positive contributions. We thus get a 'cap' before reaching $r=0$, whether we have rotation or not. We expect that a similar situation will hold for 3-charge states, and that the naive geometry will be irrelevant inside the 'horizon' radius.

Our solutions have low curvature everywhere and the dilaton is bounded; thus they are well described by their classical geometry. Note however that we do not expect the 
generic 3-charge state to be well described by a classical geometry; quantum corrections can be large. Also, while our solutions were smooth, we do not know if this will be the case for all states - the only relevant property we seek for the generic state is that the naive geometry be not valid inside a radius of the order of the naive horizon size. The generic 2-charge FP solution was singular at the location of the string, while its dual D1-D5 system was smooth for generic configurations. The singularity at the location of the $\mathrm{F}$ string changed under duality to the coordinate singularity at the center of a ' $\mathrm{KK}$ monopole tube' - the geometry in the 'cap' region is a KK monopole $\times S^{1}[5$. Generically this $S^{1}$ does not self-intersect, but if we look at a special limit where the $S^{1}$ runs $m$ times around the same path before closing then we get the conical defect singularity arising from the coincidence of $m$ KK monopoles [14, 15, 15. For the 3-charge solutions constructed in this paper we can perform dualities to interchange the D1 and P charges: $n_{1} \leftrightarrow n_{p}$. Now we have $n_{p} \neq n(n+1) n_{1} n_{5}$, so we do not get the smooth solutions constructed above; we get instead extremal limits of the general solution (3.6) which have conical defects just like the conical defects in [14, 15]. We hope to return to an analysis of more general 3-charge solutions elsewhere.

\section{Acknowledgments}

S.G. was supported by an I.N.F.N. fellowship. The work of S.D.M was supported in part by DOE grant DE-FG02-91ER-40690. We thank Oleg Lunin and Yogesh Srivastava for helpful discussions.

\section{Appendix A: The Non-Extremal Solution}

We start with a neutral rotating black hole in 5-D lifted to 10-D

$$
\begin{aligned}
d s^{2} & =-\left(1-\frac{M}{f}\right) d t^{2}+\frac{r^{2} f d r^{2}}{\left(r^{2}+a_{1}^{2}\right)\left(r^{2}+a_{2}^{2}\right)-M r^{2}}+f d \theta^{2} \\
& +\left[r^{2}+a_{1}^{2}\left(1+\frac{M}{f} \cos ^{2} \theta\right)\right] \cos ^{2} \theta d \psi^{2}+\frac{2 M a_{1} a_{2}}{f} \sin ^{2} \theta \cos ^{2} \theta d \psi d \phi \\
& +\left[r^{2}+a_{2}^{2}\left(1+\frac{M}{f} \sin ^{2} \theta\right)\right] \sin ^{2} \theta d \phi^{2}+\frac{2 M}{f} d t\left(a_{1} \cos ^{2} \theta d \psi+a_{2} \sin ^{2} \theta d \phi\right) \\
& +d y^{2}+\sum_{i=1}^{4} d z_{i}^{2}
\end{aligned}
$$

where

$$
f=r^{2}+a_{1}^{2} \sin ^{2} \theta+a_{2}^{2} \cos ^{2} \theta
$$


The parameters $a_{1}, a_{2}$ give the two angular momenta. The dilaton and the gauge fields vanish. We generate momentum along $y$ by boosting

$$
\begin{aligned}
t & =t^{\prime} \cosh \delta_{5}-y^{\prime} \sinh \delta_{5} \\
y & =-t^{\prime} \sinh \delta_{5}+y^{\prime} \cosh \delta_{5}
\end{aligned}
$$

and perform a T-duality $T_{y}$ along $y$. We get the geometry of D1 branes

$$
\begin{aligned}
d s_{10}^{2} & =-\left(1-\frac{M}{f}\right) \frac{d t^{2}}{1+K_{5}}+\frac{2 M \cosh \delta_{5}}{f\left(1+K_{5}\right)} \omega d t+\frac{d y^{2}}{1+K_{5}} \\
& +\frac{r^{2} f d r^{2}}{\left(r^{2}+a_{1}^{2}\right)\left(r^{2}+a_{2}^{2}\right)-M r^{2}}+f d \theta^{2} \\
& +\left[r^{2}+a_{1}^{2}\left(1+\frac{M}{f\left(1+K_{5}\right)} \cos ^{2} \theta\right)\right] \cos ^{2} \theta d \psi^{2}+\frac{M a_{1} a_{2} \sin ^{2} 2 \theta}{2 f\left(1+K_{5}\right)} d \psi d \phi \\
& +\left[r^{2}+a_{2}^{2}\left(1+\frac{M}{f\left(1+K_{5}\right)} \sin ^{2} \theta\right)\right] \sin ^{2} \theta d \phi^{2}+\sum_{i=1}^{4} d z_{i}^{2} \\
B_{2} & =-\frac{M \sinh \delta_{5}}{f\left(1+K_{5}\right)}\left[\cosh \delta_{5} d t+\omega\right] \wedge d y \\
e^{2 \Phi} & =\left(1+K_{5}\right)^{-1}
\end{aligned}
$$

where

$$
\omega \equiv a_{1} \cos ^{2} \theta d \psi+a_{2} \sin ^{2} \theta d \phi, \quad K_{5}=\frac{M \sinh ^{2} \delta_{5}}{f}
$$

We again boost along $y$ with parameter $S^{1}$, then do an S-duality, followed by the T-dualities $T_{1234}$. We get the D5-P solution

$$
\begin{aligned}
d s_{10}^{2} & =\sqrt{1+K_{5}}\left[-\left(1-\frac{M \cosh ^{2} \delta_{1}}{f}\right) \frac{d t^{2}}{1+K_{5}}+\left(1+\frac{M \sinh ^{2} \delta_{1}}{f}\right) \frac{d y^{2}}{1+K_{5}}\right. \\
& +\frac{2 M \cosh \delta_{5} \cosh \delta_{1}}{f\left(1+K_{5}\right)} \omega d t-\frac{M \sinh 2 \delta_{1}}{f\left(1+K_{5}\right)} d t d y-\frac{2 M \cosh \delta_{5} \sinh \delta_{1}}{f\left(1+K_{5}\right)} \omega d y \\
& +\frac{r^{2} f d r^{2}}{\left(r^{2}+a_{1}^{2}\right)\left(r^{2}+a_{2}^{2}\right)-M r^{2}}+f d \theta^{2} \\
& +\left[r^{2}+a_{1}^{2}\left(1+\frac{M}{f\left(1+K_{5}\right)} \cos ^{2} \theta\right)\right] \cos ^{2} \theta d \psi^{2}+\frac{M a_{1} a_{2} \sin ^{2} 2 \theta}{2 f\left(1+K_{5}\right)} d \psi d \phi \\
& \left.+\left[r^{2}+a_{2}^{2}\left(1+\frac{M}{f\left(1+K_{5}\right)} \sin ^{2} \theta\right)\right] \sin ^{2} \theta d \phi^{2}+\left(1+K_{5}\right)^{-1} \sum_{i=1}^{4} d z_{i}^{2}\right] \\
C_{6} & =-\frac{M \sinh \delta_{5}}{f\left(1+K_{5}\right)}\left[\cosh \delta_{5} d t \wedge d y+\omega \wedge\left(-d t \sinh \delta_{1}+d y \cosh \delta_{1}\right)\right] \wedge d z_{1} \wedge \cdots \wedge d z_{4} \\
e^{2 \Phi} & =1+K_{5}
\end{aligned}
$$


The solution above has a non-zero six-form RR field which should be converted to a two-form field before we can perform the next S-duality. The dual two form field $C_{2}$ is defined by the equation

$$
F_{3}=* F_{7}
$$

where $*$ is taken with respect to the metric above (in the string frame) and $F_{3}=d C_{2}, F_{7}=$ $d C_{6}$ are the respective field strengths. $F_{7}$ has the following non-zero components

$$
\begin{aligned}
& F_{r t y 1234}=\frac{M r \sinh 2 \delta_{5}}{f_{5}^{2}} \quad F_{\theta t y 1234}=\frac{M\left(a_{1}^{2}-a_{2}^{2}\right) \sinh 2 \delta_{5}}{2 f_{5}^{2}} \sin 2 \theta \\
& F_{r \psi t 1234}=-\frac{2 M r a_{1} \sinh \delta_{5} \sinh \delta_{1}}{f_{5}^{2}} \cos ^{2} \theta \quad F_{r \phi t 1234}=-\frac{2 M r a_{2} \sinh \delta_{5} \sinh \delta_{1}}{f_{5}^{2}} \sin ^{2} \theta \\
& F_{r \psi y 1234}=\frac{2 M r a_{1} \sinh \delta_{5} \cosh \delta_{1}}{f_{5}^{2}} \cos ^{2} \theta \quad F_{r \phi y 1234}=\frac{2 M r a_{2} \sinh \delta_{5} \cosh \delta_{1}}{f_{5}^{2}} \sin ^{2} \theta \\
& F_{\theta \psi t 1234}=-\frac{M a_{1} \sinh \delta_{5} \sinh \delta_{1}}{f_{5}^{2}}\left(r^{2}+a_{1}^{2}+M \sinh ^{2} \delta_{5}\right) \sin 2 \theta \\
& F_{\theta \phi t 1234}=\frac{M a_{2} \sinh \delta_{5} \sinh \delta_{1}}{f_{5}^{2}}\left(r^{2}+a_{2}^{2}+M \sinh ^{2} \delta_{5}\right) \sin 2 \theta \\
& F_{\theta \psi y 1234}=-\frac{M a_{1} \sinh \delta_{5} \cosh \delta_{1}}{f_{5}^{2}}\left(r^{2}+a_{1}^{2}+M \sinh ^{2} \delta_{5}\right) \sin 2 \theta \\
& F_{\theta \phi y 1234}=\frac{M a_{2} \sinh \delta_{5} \cosh \delta_{1}}{f_{5}^{2}}\left(r^{2}+a_{2}^{2}+M \sinh ^{2} \delta_{5}\right) \sin 2 \theta
\end{aligned}
$$

where

$$
f_{5} \equiv f\left(1+K_{5}\right)=r^{2}+a_{1}^{2} \sin ^{2} \theta+a_{2}^{2} \cos ^{2} \theta+M \sinh ^{2} \delta_{5}
$$

The determinant of the metric is

$$
\sqrt{-\operatorname{Det}(G)}=\frac{r f \sin \theta \cos \theta}{\sqrt{1+K_{5}}}
$$

The non-zero components of the $F_{3}$ are found to be

$$
\begin{aligned}
F_{\theta \psi \phi} & =\frac{M\left(r^{2}+a_{1}^{2}\right)\left(r^{2}+a_{2}^{2}\right) \sin 2 \theta}{f^{2}} \sinh \delta_{5} \cosh \delta_{5} \\
F_{\theta y \psi} & =\frac{M a_{2}\left(r^{2}+a_{1}^{2}\right) \sin 2 \theta}{f^{2}} \sinh \delta_{5} \sinh \delta_{1} \\
F_{\theta y \phi} & =-\frac{M a_{1}\left(r^{2}+a_{2}^{2}\right) \sin 2 \theta}{f^{2}} \sinh \delta_{5} \sinh \delta_{1} \\
F_{\theta t \psi} & =-\frac{M a_{2}\left(r^{2}+a_{1}^{2}\right) \sin 2 \theta}{f^{2}} \sinh \delta_{5} \cosh \delta_{1} \\
F_{\theta t \phi} & =\frac{M a_{1}\left(r^{2}+a_{2}^{2}\right) \sin 2 \theta}{f^{2}} \sinh \delta_{5} \cosh \delta_{1}
\end{aligned}
$$




$$
\begin{aligned}
F_{r \psi \phi} & =-\frac{M r\left(a_{1}^{2}-a_{2}^{2}\right) \sin ^{2} 2 \theta}{2 f^{2}} \sinh \delta_{5} \cosh \delta_{5} \\
F_{r y \psi} & =\frac{2 M r a_{2} \cos ^{2} \theta}{f^{2}} \sinh \delta_{5} \sinh \delta_{1} \\
F_{r y \phi} & =\frac{2 M r a_{1} \sin ^{2} \theta}{f^{2}} \sinh \delta_{5} \sinh \delta_{1} \\
F_{r t \psi} & =-\frac{2 M r a_{2} \cos ^{2} \theta}{f^{2}} \sinh \delta_{5} \cosh \delta_{1} \\
F_{r t \phi} & =-\frac{2 M r a_{1} \sin ^{2} \theta}{f^{2}} \sinh \delta_{5} \cosh \delta_{1}
\end{aligned}
$$

For the above field strength we find the following gauge field ${ }^{2}$

$$
\begin{aligned}
C_{\psi \phi} & =-\frac{M\left(r^{2}+a_{2}^{2}\right) \cos ^{2} \theta}{f} \sinh \delta_{5} \cosh \delta_{5} \\
C_{y \psi} & =-\frac{M a_{2} \cos ^{2} \theta}{f} \sinh \delta_{5} \sinh \delta_{1}, \quad C_{y \phi}=-\frac{M a_{1} \sin ^{2} \theta}{f} \sinh \delta_{5} \sinh \delta_{1} \\
C_{t \psi} & =\frac{M a_{2} \cos ^{2} \theta}{f} \sinh \delta_{5} \cosh \delta_{1}, \quad C_{t \phi}=\frac{M a_{1} \sin ^{2} \theta}{f} \sinh \delta_{5} \cosh \delta_{1}
\end{aligned}
$$

Performing an S-duality followed by $T_{y}$ we get the F1-NS5 solution

$$
\begin{aligned}
d s^{2} & =-\left(1-\frac{M}{f}\right)\left(1+K_{1}\right)^{-1} d t^{2}+\left(1+K_{1}\right)^{-1} d y^{2} \\
& +f\left(1+K_{5}\right)\left[\frac{\left.r^{2}\right) d r^{2}}{\left(r^{2}+a_{1}^{2}\right)\left(r^{2}+a_{2}^{2}\right)-M r^{2}}+d \theta^{2}\right] \\
& +\left[\left(r^{2}+a_{1}^{2}\right)\left(1+K_{5}\right)+\frac{M a_{1}^{2} \cos ^{2} \theta}{f\left(1+K_{1}\right)}+\left(a_{2}^{2}-a_{1}^{2}\right) \frac{K_{5} K_{1}}{1+K_{1}} \cos ^{2} \theta\right] \cos ^{2} \theta d \psi^{2} \\
& +\left[\left(r^{2}+a_{2}^{2}\right)\left(1+K_{5}\right)+\frac{M a_{2}^{2} \sin ^{2} \theta}{f\left(1+K_{1}\right)}+\left(a_{1}^{2}-a_{2}^{2}\right) \frac{K_{5} K_{1}}{1+K_{1}} \sin ^{2} \theta\right] \sin ^{2} \theta d \phi^{2} \\
& +\frac{2 M a_{1} a_{2} \sin ^{2} \theta \cos ^{2} \theta}{f\left(1+K_{1}\right)} d \psi d \phi+\frac{2 M a_{1} \cosh \delta_{5} \cosh \delta_{1}}{f\left(1+K_{1}\right)} \cos ^{2} \theta d \psi d t \\
& +\frac{2 M a_{2} \cosh \delta_{5} \cosh \delta_{1}}{f\left(1+K_{1}\right)} \sin ^{2} \theta d \phi d t+\frac{2 M a_{2} \sinh \delta_{5} \sinh \delta_{1}}{f\left(1+K_{1}\right)} \cos ^{2} \theta d \psi d y \\
& +\frac{2 M a_{1} \sinh \delta_{5} \sinh \delta_{1}}{f\left(1+K_{1}\right)} \sin ^{2} \theta d \phi d y+\sum_{i=1}^{4} d z_{i}^{2}
\end{aligned}
$$

${ }^{2}$ This choice is up to the usual gauge freedom. In particular the apparent asymmetry between $a_{1}$ and $a_{2}$ in the component $C_{\psi \phi}$ can be cured by a different gauge choice. 


$$
\begin{aligned}
B_{2} & =\frac{M \cos ^{2} \theta}{f\left(1+K_{1}\right)}\left(a_{2} \sinh \delta_{5} \cosh \delta_{1} d t+a_{1} \cosh \delta_{5} \sinh \delta_{1} d y\right) \wedge d \psi \\
& +\frac{M \sin ^{2} \theta}{f\left(1+K_{1}\right)}\left(a_{1} \sinh \delta_{5} \cosh \delta_{1} d t+a_{2} \cosh \delta_{5} \sinh \delta_{1} d y\right) \wedge d \phi \\
& -\frac{M \sinh \delta_{1} \cosh \delta_{1}}{f\left(1+K_{1}\right)} d t \wedge d y-\frac{M \sinh 2 \delta_{5}}{2 f\left(1+K_{1}\right)}\left(r^{2}+a_{2}^{2}+M \sinh ^{2} \delta_{1}\right) \cos ^{2} \theta d \psi \wedge d \phi \\
e^{2 \Phi} & =\frac{1+K_{5}}{1+K_{1}}
\end{aligned}
$$

Finally, doing an S-duality and performing a boost with parameter $\delta_{p}$ we get the D1-D5-P solution (4.21)-(4.23).

\section{References}

[1] S. W. Hawking, Commun. Math. Phys. 43, 199 (1975).

[2] S. D. Mathur, Nucl. Phys. B 529, 295 (1998) arXiv:hep-th/9706151.

[3] O. Lunin and S. D. Mathur, Nucl. Phys. B 623, 342 (2002) arXiv:hep-th/0109154.

[4] O. Lunin and S. D. Mathur, Phys. Rev. Lett. 88, 211303 (2002) arXiv:hep-th/0202072.

[5] O. Lunin, J. Maldacena and L. Maoz, arXiv:hep-th/0212210.

[6] A. Strominger and C. Vafa, Phys. Lett. B 379, 99 (1996) arXiv:hep-th/9601029.

[7] S. D. Mathur, A. Saxena and Y. K. Srivastava, Nucl. Phys. B 680, 415 (2004) arXiv:hep-th/0311092.

[8] O. Lunin, arXiv:hep-th/0404006.

[9] J. B. Gutowski, D. Martelli and H. S. Reall, Class. Quant. Grav. 20, 5049 (2003) arXiv:hep-th/0306235.

[10] N. Seiberg and E. Witten, JHEP 9904, 017 (1999) arXiv:hep-th/9903224; F. Larsen and E. J. Martinec, JHEP 9906, 019 (1999) arXiv:hep-th/9905064|; J. de Boer, Nucl. Phys. B 548, 139 (1999) arXiv:hep-th/9806104.

[11] O. Lunin and S. D. Mathur, Commun. Math. Phys. 219, 399 (2001) arXiv:hep-th/0006196.

[12] O. Lunin and S. D. Mathur, Commun. Math. Phys. 227, 385 (2002) arXiv:hep-th/0103169.

[13] A. Schwimmer and N. Seiberg, Phys. Lett. B 184, 191 (1987). 
[14] V. Balasubramanian, J. de Boer, E. Keski-Vakkuri and S. F. Ross, Phys. Rev. D 64, 064011 (2001) arXiv:hep-th/0011217.

[15] J. M. Maldacena and L. Maoz, JHEP 0212, 055 (2002) arXiv:hep-th/0012025.

[16] M. Cvetic and D. Youm, Nucl. Phys. B 476, 118 (1996) arXiv:hep-th/9603100; D. Youm, Phys. Rept. 316, 1 (1999) arXiv:hep-th/9710046; M. Cvetic and F. Larsen, Nucl. Phys. B 531, 239 (1998) arXiv:hep-th/9805097.

[17] J. C. Breckenridge, R. C. Myers, A. W. Peet and C. Vafa, Phys. Lett. B 391, 93 (1997) arXiv:hep-th/9602065.

[18] C. A. R. Herdeiro, Nucl. Phys. B 582, 363 (2000) arXiv:hep-th/0003063; C. A. R. Herdeiro, Nucl. Phys. B 665, 189 (2003) arXiv:hep-th/0212002.

[19] L. Dyson, JHEP 0403, 024 (2004) arXiv:hep-th/0302052.

[20] H. Elvang and R. Emparan, JHEP 0311, 035 (2003) arXiv:hep-th/0310008.

[21] I. Bena and P. Kraus, arXiv:hep-th/0402144.

[22] I. Bena, arXiv:hep-th/0404073.

[23] B. C. Palmer and D. Marolf, arXiv:hep-th/0403025.

[24] R. Emparan, JHEP 0403, 064 (2004) arXiv:hep-th/0402149.

[25] D. Mateos and P. K. Townsend, Phys. Rev. Lett. 87, 011602 (2001) arXiv:hep-th/0103030; R. Emparan, D. Mateos and P. K. Townsend, JHEP 0107, 011 (2001) arXiv:hep-th/0106012.

[26] R. Gopakumar and C. Vafa, Adv. Theor. Math. Phys. 3, 1415 (1999) arXiv:hep-th/9811131|; J. M. Maldacena and C. Nunez, Phys. Rev. Lett. 86, 588 (2001) arXiv:hep-th/0008001|; A. Iqbal, N. Nekrasov, A. Okounkov and C. Vafa, arXiv:hep-th/0312022. 